\title{
Singularities in the Image-based Visual Servoing of Five Points
}

\author{
Abhilash Nayak and Sbastien Briot
}

\begin{abstract}
Image-based Visual Servoing (IBVS) hinges on the observation of geometric entities such as 3D points on a target object and their projected image on the camera to control a robot. It includes a mapping between two vector spaces: the time variation of the projected $2 \mathrm{D}$ points on the image plane of the camera and the instantaneous spatial velocity of the camera induced by the motion of the robot. The configuration of the camera and the points is said to be singular if the matrix that maps the two vector spaces, the so-called interaction matrix, loses rank. Utilizing the results on singularities in visual servoing of three and four $3 \mathrm{D}$ points, the goal of this article is to determine the configuration of five points leading to singularities. The problem boils down to examining the vanishing conditions of all the minors of the interaction matrix. It turns out that it is not necessary to consider all the minors. Thus, the simplest set of equations is considered and tools from computational algebraic geometry are used to derive a necessary condition leading to singularities in IBVS of five points.
\end{abstract}

Key words: IBVS, interaction matrix, singularities, algebraic geometry.

\section{Introduction}

Visual servoing is the closed-loop position control of a robot using vision. A particular technique relevant to this article is when a camera is attached to the endeffector (EE) of a robot, called as the eye-in-hand configuration [5]. Thus, the motion of the robot induces the motion of the camera which obtains the vision data from the object to control the robot. One could look at the features drawn on the object such as points, lines or curves to relatively position the EE w.r.t their pro-

Abhilash Nayak and Sbastien Briot,

CNRS, Laboratoire des Sciences du Numérique de Nantes (LS2N), Nantes, France. e-mail: Abhilash.Nayak@1s2n.fr, Sebastien.Briot@1s2n.fr 
jection in the camera space $[2,3,8]$. This kind of control which depends on the use of these geometric entities as perceived by the camera is called Image-Based Visual Servoing $(I B V S)$. A concise account of basic and advanced approaches in IBVS can be found in Chaumette and Hutchinson [4,5]. Essentially, the control of the robot is based on the rate of change of the image observed by the vision system induced by the motion of the manipulator EE. There could be instances when the image observed by the vision system is stationary although the EE is moving, which leads to controllability issues. They are called singularities in the IBVS $[4,5]$.

Although it is crucial to determine these singularities, there exists hardly any research in this regard due to the complexity of the mathematical problem. The singularities in the observation of three 3D points were first identified geometrically in Michel and Rives [13]. Recently, we have algebraically proved that there are always a finite set of singular positions of the camera given four non-degenerate points [1]. Therefore, the goal of this article is to take it a step further and to determine the conditions for the occurrence of singularities when five non-degenerate 3D points are observed.

\section{Interaction matrix corresponding to IBVS of points}

In the context of this article, the set of features observed on an object used to control the robot are points. The corresponding interaction matrix is recalled in this section. Following the standard pin-hole camera model with a focal length of 1 unit shown in Fig. 1, let the camera center $C$ be at the origin of the EE coordinate frame. Let the camera axis be along the $z$-axis and the image plane be parallel to the $x y$-plane. For a point $M(x, y, z)$ expressed in the EE coordinate frame, the mapping between EE twist (i.e. $\mathbf{v}_{c}=\left[v_{c}, \omega_{c}\right]^{T}$, where $\mathbf{v}$ is the instantaneous linear velocity of the optical center $C$ and $\omega$ is the instantaneous angular velocity of the camera) and the rate of change of image features on the image plane (i.e. $\dot{\mathbf{s}}=[\dot{u}, \dot{v}]^{T}$, where $(u, v)$ are the coordinates of the projection of $M$ onto the image plane) in Fig. 1 is expressed as follows $[6,11]$ :

$$
\dot{\mathbf{s}}=\mathbf{L}(\mathbf{s}) \mathbf{v}_{c}, \quad\left[\begin{array}{c}
\dot{u} \\
\dot{v}
\end{array}\right]=\left[\begin{array}{cccccc}
-z & 0 & x & x y & -x^{2}-z^{2} & y z \\
0 & -z & y & y^{2}+z^{2} & -x y & -z x
\end{array}\right]\left[\begin{array}{c}
v_{c} \\
\omega_{c}
\end{array}\right] .
$$

It should be noted that unlike in this article, a standard way to express the interaction matrix is in terms of $u, v$ and the depth $z$. Although the final results should be identi$\mathrm{cal}$, the results with the current parametrization are easier to interpret geometrically. For $n$-points, $\mathbf{L}$ is a $(2 n \times 6)$ matrix formed by the concatenation of rows of the matrix in Eq. (1). Henceforth, the dimension of a matrix is written as its subscript. The singular configurations in IBVS of $n$-points correspond to the cases in which $\mathbf{L}_{(2 n \times 6)}$ has a non-zero kernel [11]. To control 6 degrees of freedom of a robot, it is necessary to observe at least three $3 \mathrm{D}$ points. However, in this case, the pose of the camera in the control scheme is not unique [4]. As a result, generally, more 


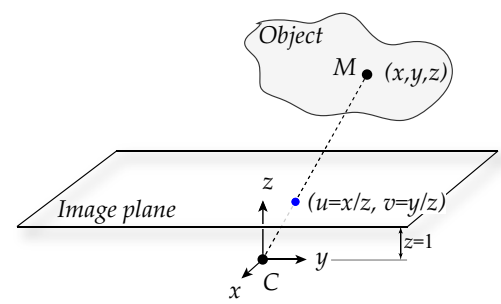

Fig. 1 The pin-hole camera model

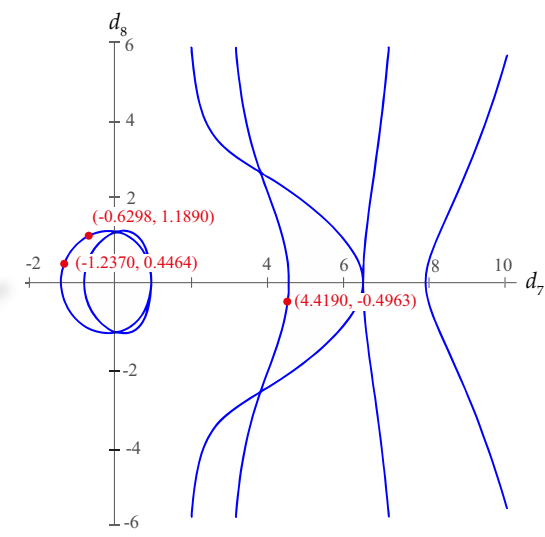

Fig. $2 V(\mathscr{K}) \in V($ res $)$, only three elements of $V(\mathscr{K})$ in Eq. (16) are shown for clarity.

than three points are observed. It has been established that for three non-coplanar points, $\mathbf{L}_{(6 \times 6)}$ is rank deficient when the camera center lies on an affine cylinder that passes through the three points with its axis normal to the plane containing the points [13]. For four non-degenerate (of which no three points are collinear nor all four coplanar) points, it was shown in [1] that any configuration of them leads to a finite number of singular positions of the camera.

The goal is to find the configurations of five non-degenerate points and the camera center such that $\operatorname{rank}\left(\mathbf{L}_{(10 \times 6)}\right)<6$.

\section{Algebraic description of the problem}

Without any loss of generality, the affine coordinates of the five points in the object coordinate frame can be considered as follows:

$$
\begin{array}{ll}
M_{1}=[0,0,0]^{T}, & M_{2}=[1,0,0]^{T}, \quad M_{3}=\left[d_{1}, d_{2}, 0\right]^{T}, \\
M_{4}=\left[d_{3}, d_{4}, d_{5}\right]^{T}, & M_{5}=\left[d_{6}, d_{7}, d_{8}\right]^{T} .
\end{array}
$$

Let the coordinates of the camera expressed in the object frame be $C=[x, y, z]^{T}$. The orientation between the object and the camera coordinate frames is assumed constant. This is due to the fact that the relative configuration of the points and the camera center are invariant to rotations. For any other object orientation given by a rotation matrix $\mathbf{R}$, the singularities can be determined by rotating the singular position coordinates of the camera obtained for the corresponding fixed orientation by $\mathbf{R}$ as proven in [1,2].

If only the first three points are considered (with $d_{2} \neq 0$ ), the singular positions of the camera correspond to the vanishing determinant of $\mathbf{L}_{(6 \times 6)}$ [6]: 


$$
C_{123}:=x^{2} d_{2}-x d_{2}+y^{2} d_{2}+\left(-d_{1}^{2}-d_{2}^{2}+d_{1}\right) y=0 .
$$

It is the equation of a cylinder in $\mathbb{R}[x, y, z]$ with its cross-section being the circumcircle of $\triangle M_{1} M_{2} M_{3}$ and axis normal to the plane containing $M_{1}, M_{2}$ and $M_{3}$ [13].

In case of four points, the interaction matrix is no longer square. Hence, the singular positions of the camera correspond to the vanishing of all of the $(8 \times 6)$ minors of $\mathbf{L}_{(8 \times 6)}$ [1]. There are 28 minors and they are polynomials in variables $x, y, z$ and parameters $d_{1}, d_{2}, d_{3}, d_{4}, d_{5}$. This set of polynomials generates a polynomial ideal $\mathscr{L}_{4} \subset \mathbb{R}\left[x, y, z, d_{1}, d_{2}, d_{3}, d_{4}, d_{5}\right]$. It is an infinite set that consists of all combinations of those minors with coefficients chosen from $\mathbb{R}\left[x, y, z, d_{1}, d_{2}, d_{3}, d_{4}, d_{5}\right]$ and is denoted by angular brackets ${ }^{1}$. As shown in [1], four of the minors must be singular cylinders corresponding to the four sets of three points. Let them generate an ideal

$$
\mathscr{C}_{4}=\left\langle C_{123}, C_{124}, C_{134}, C_{234}\right\rangle \subset \mathbb{R}\left[x, y, z, d_{1}, d_{2}, d_{3}, d_{4}, d_{5}\right],
$$

where $C_{i j k}$ is the cylinder of singularities in $\mathbb{R}[x, y, z]$ corresponding to points $M_{i}, M_{j}$ and $M_{k}$. The sets of common zeros to the minors correspond to the so called varieties of the ideals, denoted as $V\left(\mathscr{L}_{4}\right)$ and $V\left(\mathscr{C}_{4}\right)$, respectively. Geometrically, $V\left(\mathscr{C}_{4}\right)$ is the intersection of the four cylinders $C_{123}, C_{124}, C_{134}, C_{234}$ and $V\left(\mathscr{C}_{4}\right) \supseteq V\left(\mathscr{L}_{4}\right)$. It was shown in [1] that when $d_{2} \neq 0$ and $d_{5} \neq 0, V\left(\mathscr{L}_{4}\right)=V\left(\mathscr{C}_{4}\right)$. Furthermore, it was also proved that there are at least 2 and at most 6 singular positions of the camera for any given non-degenerate configuration of four points.

The interest of the current article is to explore the outcome of adding a fifth point on the singular positions of the camera. In this vein, the $(10 \times 6)$ interaction matrix $\mathbf{L}_{(10 \times 6)}$ obtained when observing five points has 210 principal minors that generate the ideal $\mathscr{L}_{5} \subset \mathbb{R}\left[x, y, z, d_{i}, i=1, \ldots, 8\right]$. Among these minors, 10 cylinders corresponding to the singularities of 10 sets of three points can be picked out to generate the ideal

$$
\mathscr{C}_{5}=\left\langle C_{123}, C_{124}, C_{125}, C_{134}, C_{135}, C_{145}, C_{234}, C_{235}, C_{245}, C_{345}\right\rangle \subset \mathbb{R}\left[x, y, z, d_{i}\right] .
$$

Unlike for four points, the computational software such as Maple and Singular fail to prove that $V\left(\mathscr{L}_{5}\right)=V\left(\mathscr{C}_{5}\right)$ for any $d_{i}, i=1, \ldots, 8$, due to the large number of variables and equations. However, since the intersection of 10 cylinders is the largest variety (i.e. $V\left(\mathscr{C}_{5}\right) \supseteq V\left(\mathscr{L}_{5}\right)$ ), it will be considered for further analysis in section 4.2 (not in 4.1).

\footnotetext{
${ }^{1}$ The terms from algebraic geometry are defined briefly in the context of this article as and when they are used. For formal definitions and examples, the reader is directed to the excellent book by Cox, Little and O'Shea [7].
} 


\section{Relative configuration of five points for the occurrence of singularities}

It is clear by now that starting from three points, addition of a point to be observed decreases the dimension of the singular manifold (cylinder in case of three points, a finite set of points in case of four points) for the placement of the camera [10]. So, it is expected that adding a fifth point should in general not lead to any singularities. However, it is possible to choose a fifth point in such a way that the position of the camera coincides with the singularities corresponding to the first four points. This section aims at determining these relations between $d_{i}, i=1, \ldots, 8$ such that the 10 cylinders in Eq. (4) have a non-zero intersection. This is shown initially by fixing some of the $d_{i}$ values. Subsequently, a necessary condition with arbitrary $d_{i}$ values is derived. All the calculations done in this section can be found in the Maple sheet [12].

\subsection{First four points are fixed}

In this sub-section, the coordinates of the five points are chosen arbitrarily as follows:

$$
\left\{d_{1}=2, d_{2}=7, d_{3}=5, d_{4}=-6, d_{5}=4, d_{6}=3, d_{7}=-11, d_{8}=13\right\}
$$

When all five points are fixed according to (5), the Gröbner basis (set of generators of an ideal such that it has a nicer structure and one of its applications is to solve a set of polynomial equations [7]) of ideal $\mathscr{L}_{5}$ yields $\langle 1\rangle$ implying:

$$
V\left(\mathscr{L}_{5}\right)=\emptyset \quad \text { for } \quad \mathscr{L}_{5} \subset \mathbb{R}[x, y, z]
$$

In other words, for a set of non-degenerate $\left(d_{2}, d_{5}, d_{8} \neq 0\right.$ and so on) five points, there are no singular positions of the camera. Therefore, the conditions for $V\left(\mathscr{L}_{5}\right) \neq \emptyset$ is explored in the following by letting the parameters $d_{i}$ arbitrary one at a time. If $d_{8}$ is not substituted, the solution set is still empty. However, if both $d_{7}$ and $d_{8}$ are left arbitrary (i.e. $\mathscr{L}_{5} \subset \mathbb{R}\left[d_{7}, d_{8}, x, y, z\right]$ ), the solution set is finite:

$$
\begin{aligned}
V\left(\mathscr{L}_{5}\right)=\left\{d_{7}\right. & =4.4190, d_{8}=-.4963, x=3.4390, y=5.8530, z=25.1100 \\
& d_{7}=-1.2730, d_{8}=.4464, x=3.4390, y=5.8530, z=25.1100 \\
& d_{7}=7.4800, d_{8}=24.7200, x=3.4390, y=5.8530, z=25.1100 \\
& \left.d_{7}=-.6298, d_{8}=1.1890, x=-2.4390, y=1.4330, z=3.8180\right\}
\end{aligned}
$$

The above set gives the placement of the fifth point and the camera such that there are singularities in the IBVS of five points. Furthermore, if $M_{5}$ is left arbitrary, the dimension of the following variety is 1 : 


$$
\operatorname{dim}\left(V\left(\mathscr{L}_{5}\right)\right)=1 \quad \text { for } \quad \mathscr{L}_{5} \subset \mathbb{R}\left[d_{6}, d_{7}, d_{8}, x, y, z\right] .
$$

Therefore, if the first four points are fixed, there exists a curve on which the fifth point lies such that the the singularities appear in the IBVS of five points. To obtain the equations describing this curve, $x, y, z$ must be eliminated. This amounts to computing the elimination ideal (Geometrically, it amounts to projecting the corresponding variety onto a lower dimensional subspace):

$$
\mathscr{K}_{s}=\mathscr{L}_{5} \cap \mathbb{R}\left[d_{6}, d_{7}, d_{8}\right] .
$$

$\mathscr{K}_{s}$ is generated by 9 polynomials that are functions of only $d_{6}, d_{7}$ and $d_{8}$. Recall that this ideal is calculated when the first four points are fixed according to (5). On the other hand, due to the complexity of the equations, Maple fails to calculate this ideal symbolically (when all the points are arbitrary):

$$
\mathscr{K}=\mathscr{L}_{5} \cap \mathbb{R}\left[d_{i}\right], i=1, \ldots, 8,
$$

where $\mathscr{K}$ is the ideal obtained after eliminating $x, y, z$ from polynomials generating $\mathscr{L}_{5}$. However, a workaround is proposed in the next sub-section to obtain a necessary relation between the parameters $d_{i}, i=1, \ldots, 8$ that leads to singularities. Nonetheless, obtaining necessary and sufficient relation(s) between the parameters $d_{i}$ is analogous to finding the elimination ideal in Eq. (10) and is still an open question. Some ideas to obtain it are discussed in the section on conclusions.

\subsection{All five points are chosen arbitrarily}

The goal of this sub-section is to come up with a necessary condition such that $V\left(\mathscr{L}_{5}\right) \neq \emptyset$. Since $\mathscr{L}_{5} \supseteq \mathscr{C}_{5}$ (dually $V\left(\mathscr{L}_{5}\right) \subseteq V\left(\mathscr{C}_{5}\right)$ ), it is enough to determine the necessary relations between the coordinates of five points so that the cylinders $C_{i j k}=0$ in $\mathscr{C}_{5}$ have a non-zero intersection.

It is already known from [1] that the Gröbner basis of $\mathscr{C}_{4}$, a sub-ideal of $\mathscr{C}_{5}$ in Eq. (3) yields a univariate sextic polynomial in variable $x$ and parameters $d_{i}, i=1, . ., 5$ that can be transformed to a cubic $G_{C 4}$ with a different variable $q$ :

$$
G_{C 4}=a_{3} q^{3}+a_{2} q^{2}+a_{1} q+a_{0}, a_{i} \in \mathbb{R}\left[d_{1}, d_{2}, d_{3}, d_{4}, d_{5}\right],
$$

where $q$ is an affine fucntion of $x$. Another similar sub-ideal of $\mathscr{C}_{5}$ can be considered: $\mathscr{C}_{4 b}=\left\langle C_{123}, C_{125}, C_{135}, C_{235}\right\rangle \subset \mathbb{R}\left[x, y, z, d_{1}, d_{2}, d_{6}, d_{7}, d_{8}\right]$. The varieties $V\left(\mathscr{C}_{4}\right)$ and $V\left(\mathscr{C}_{4 b}\right)$ are intersections of cylinders $C_{i j k}$ corresponding to points $M_{1}, M_{2}, M_{3}$, $M_{4}$ and $M_{1}, M_{2}, M_{3}, M_{5}$, respectively. As a consequence, simply replacing the coordinates of point $M_{4}$ by that of $M_{5}$ (i.e. $d_{3} \rightarrow d_{6}, d_{4} \rightarrow d_{7}, d_{5} \rightarrow d_{8}$ ) in $G_{C 4}$, gives another cubic polynomial $G_{C 4 b}$ in variable $q$ :

$$
G_{C 4 b}=b_{3} q^{3}+b_{2} q^{2}+b_{1} q+b_{0}, b_{i} \in \mathbb{R}\left[d_{1}, d_{2}, d_{6}, d_{7}, d_{8}\right] .
$$


Moreover, if the 10 cylinders $C_{i j k}=0$ that generate $\mathscr{C}_{5}$ in Eq. (4) share a non-zero solution set, it is necessary that the solutions lie on the 7 cylinders that belong to both the ideals $\mathscr{C}_{4}$ and $\mathscr{C}_{4 b}$ as follows [12]:

$$
\begin{aligned}
\left\langle C_{123}, C_{124}, C_{125}, C_{134}, C_{135}, C_{234}, C_{235}\right\rangle=\mathscr{C}_{4} \cup \mathscr{C}_{4 b} \subseteq \mathscr{C}_{5} \subseteq \mathscr{L}_{5} \\
\Longrightarrow V\left(\mathscr{C}_{4}\right) \cap V\left(\mathscr{C}_{4 b}\right) \supseteq V\left(\mathscr{C}_{5}\right) \supseteq V\left(\mathscr{L}_{5}\right)
\end{aligned}
$$

The condition for $G_{C 4}=0$ and $G_{C 4 b}=0$ to have the same solutions (so that $\left.V\left(\mathscr{C}_{5}\right), V\left(\mathscr{L}_{5}\right) \neq \emptyset\right)$ is that their resultant w.r.t $q$ must be zero. The resultant is a 58 degree polynomial in $d_{i}, i=1, \ldots, 8$.

$$
r e s=-d_{1}^{32} d_{4}^{12} d_{6}^{2} d_{7}^{4} d_{8}^{8}-2 d_{1}^{32} d_{4}^{12} d_{6}^{2} d_{7}^{2} d_{8}^{10}-d_{1}^{32} d_{4}^{12} d_{6}^{2} d_{8}^{12}+\cdots
$$

It implies that, res $=0$ is a necessary condition for $V\left(\mathscr{L}_{5}\right), V\left(\mathscr{C}_{5}\right) \neq 0$. As a result, the $d_{i}$ values satisfying the generators of $\mathscr{K}$ in Eq. (10) must also satisfy res $=0$. Dually, res must belong to the ideal $\mathscr{K}$ :

$$
\text { res } \in \mathscr{K} \Longleftrightarrow V(\mathscr{K}) \in V(\text { res })
$$

The above relation can be verified by substituting parameters $d_{i}, i=1, \ldots, 6$ from (5). The reason for substituting only first six parameters is to be able to visualize the results since in this case, $V(\mathscr{K})$ is a finite set as follows:

$$
\begin{aligned}
V(\mathscr{K})=\{(-1.2370,0.4464),(4.4190,-0.4963) \\
\\
(7.4800,24.7200),(-0.6298,1.1890)\} .
\end{aligned}
$$

It corresponds exactly to coordinates of the fifth point in (7) that lead to singularities. The inverse condition number of the matrix $\mathbf{L}_{(6 \times 10)}^{T} \mathbf{L}_{(10 \times 6)}$ for the aforementioned configuration of five points is of the range $10^{-19}$ implying they are indeed singularities in the IBVS. $V($ res $)$ or simply res $=0$ is a curve in the $d_{7} d_{8}$-plane and $V(\mathscr{K}) \in V($ res $)$ as shown in Fig. 2 on page 3. Moreover, it can be verified in Maple that res $\in \mathscr{K}$.

For practical purposes, it is enough to have a necessary condition. The five points must be chosen such that they do not satisfy the relation res $=0$, which assures that there are no singularities. However, the converse is not true, i.e. any $d_{i}, i=1, \ldots, 8$ satisfying $r e s=0$ is not sufficient for the occurrence of singularities. Therefore, to obtain necessary and sufficient conditions (It is evident from Eq. (9) that there is more than one relation) to avoid the singularities is still an open question and perhaps the symmetry of the cylinder equations holds the key.

\section{Conclusions}

Image-based visual servoing of five points is mostly free of singularities when their relative configuration is non-degenerate. However, there are some special configu- 
rations which might lead to problems in the velocity control. This article focused on determining those configurations as relations between the affine coordinates of the points. The problem was formulated as determining the set of common zeros to the principal minors of the interaction matrix. It turned out that they do not have common zeros when the five points are chosen arbitrarily. Therefore, a scenario was considered in which the first four points are fixed and conditions on the fifth point such that the minors have common zeros were derived. Additionally, using Gröbner basis computations, a necessary relation between the coordinates of all five points for the occurrence of singularities was derived .

It is noteworthy that the singular positions of the camera do not change when the fourth and the fifth points are interchanged. Using this idea, Faugre's Abelian F5 algorithm [9] will be used in the future to obtain $\mathscr{K}$ in Eq. (10).

Acknowledgements This work was supported by the French ANR project SESAME (funding ID: ANR-18-CE33-0011)

\section{References}

1. B.Pascual-Eduardo, Nayak, A., Briot, S., Kermorgant, O., Martinet, P., Chaumette, F., Din, M.S.E.: Technical report: New results on the singularity cases when observing four image points (2020). URL https://uncloud.univ-nantes.fr/index.php/s/SpoRgBwpfeCTryD

2. Briot, S., Chaumette, F., Martinet, P.: Revisiting the determination of the singularity cases in the visual servoing of image points through the concept of "hidden robot" 33(2) (2017)

3. Briot, S., Martinet, P., Chaumette, F.: Singularity cases in the visual servoing of three image lines 2(2), 412-419 (2017)

4. Chaumette, F., Hutchinson, S.: Visual servo control Part I: Basic approaches. IEEE Robotics and Automation Magazine 13(4), 82-90 (2006)

5. Chaumette, F., Hutchinson, S.: Visual servo control, Part II: Advanced approaches. IEEE Robotics and Automation Magazine 14(1), 109-118 (2007)

6. Chaumette, F., Hutchinson, S.: Visual Servoing and Visual Tracking, chapter 24 of Handbook of Robotics. Springer (2008)

7. Cox, D.A., Little, J., O’Shea, D.: Ideals, Varieties, and Algorithms: An Introduction to Computational Algebraic Geometry and Commutative Algebra, 3/e (Undergraduate Texts in Mathematics). Springer-Verlag, Berlin, Heidelberg (2007)

8. Espiau, B., Chaumette, F., Rives, P.: A new approach to visual servoing in robotics. IEEE Transactions on Robotics and Automation 8(3) (1992)

9. Faugre, J.C., Svartz, J.: Gröbner bases of ideals invariant under a commutative group: The non-modular case. In: Proceedings of the 38th International Symposium on Symbolic and Algebraic Computation, ISSAC 13, p. 347354. Association for Computing Machinery (2013)

10. Feddema, J., Lee, C., Mitchell, O.: Automatic selection of image features for visual servoing of a robot manipulator. In: Proceedings of the 1989 IEEE International Conference on Robotics and Automation (ICRA 1989), pp. 832-837. Scottsdale (1989)

11. Hutchinson, S., Hager, G., Corke, P.: A tutorial on visual servo control. IEEE Transactions on Robotics and Automation 12, 651-670 (1996)

12. Maple-file: Maple file with calculations (2020). URL https://uncloud.univnantes.fr/index.php/s/QcHAtkzQimJW278

13. Michel, H., Rives, P.: Singularities in the determination of the situation of a robot effector from the perspective view of 3 points. Tech. rep., INRIA (1993) 